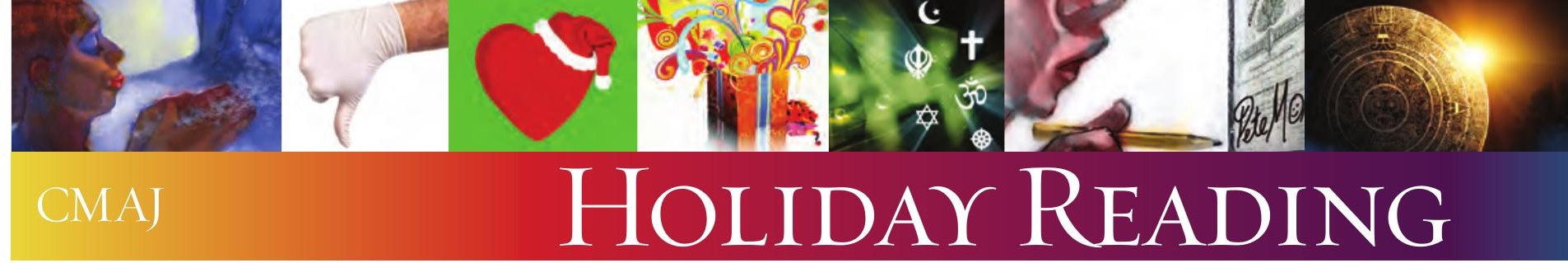

\title{
The Mayan Doomsday's effect on survival outcomes in clinical trials
}

\author{
Paul Wheatley-Price MBChB MD, Brian Hutton MSc PhD, Mark Clemons MBBS MD
}

$\mathrm{T}$

here is a great deal of speculation concerning the end of the world in December 2012, coinciding with the end of the Mesoamerican Long Count calendar (the "Maya calendar"). Such an event would undoubtedly affect population survival and, thus, survival outcomes in clinical trials. Here, we discuss how the outcomes of clinical trials may be affected by the extinction of all mankind and recommend appropriate changes to their conduct. In addition, we use computer modelling to show the effect of the apocalypse on a sample clinical trial.

\section{Mostly true background}

With the imminent completion of the Maya calendar, many "experts" suspect the end of the world will occur on Dec. 21, 2012. ${ }^{1,2}$ This impending apocalypse highlights the need to rapidly resolve many major issues in research methods. Being based in an academic institution and fully respectful and compliant with all issues concerning Good Clinical Practice guidelines, we have been told that a tenet of human research is that all clinical trials must report and disseminate statistical results in a timely manner for the benefit of patients and the wider scientific community. ${ }^{3}$ Given the anticipated effect of the apocalypse, hence known as Mayan Doomsday (MaD), on survival outcome measures, research methods must be adjusted for the expedited analysis of all clinical research currently in progress. We recognize that reviewers, editors and even readers of this article may find the premise of our commentary rather fantastical. However, we defend the importance of our opinion by quoting the great medical forefather Louis Pasteur:

Where observation is concerned, fortune favours only the prepared mind.

\section{Stuff we did}

Using computer modelling (described elsewhere ad nauseum, ad infinitum), we have generated Kaplan-Meier curves for the overall population to compare normal life expectancy from standard actuary tables (control group) with population survival after MaD (obliteration group). These methods were then hypothetically applied to a standard clinical trial design.

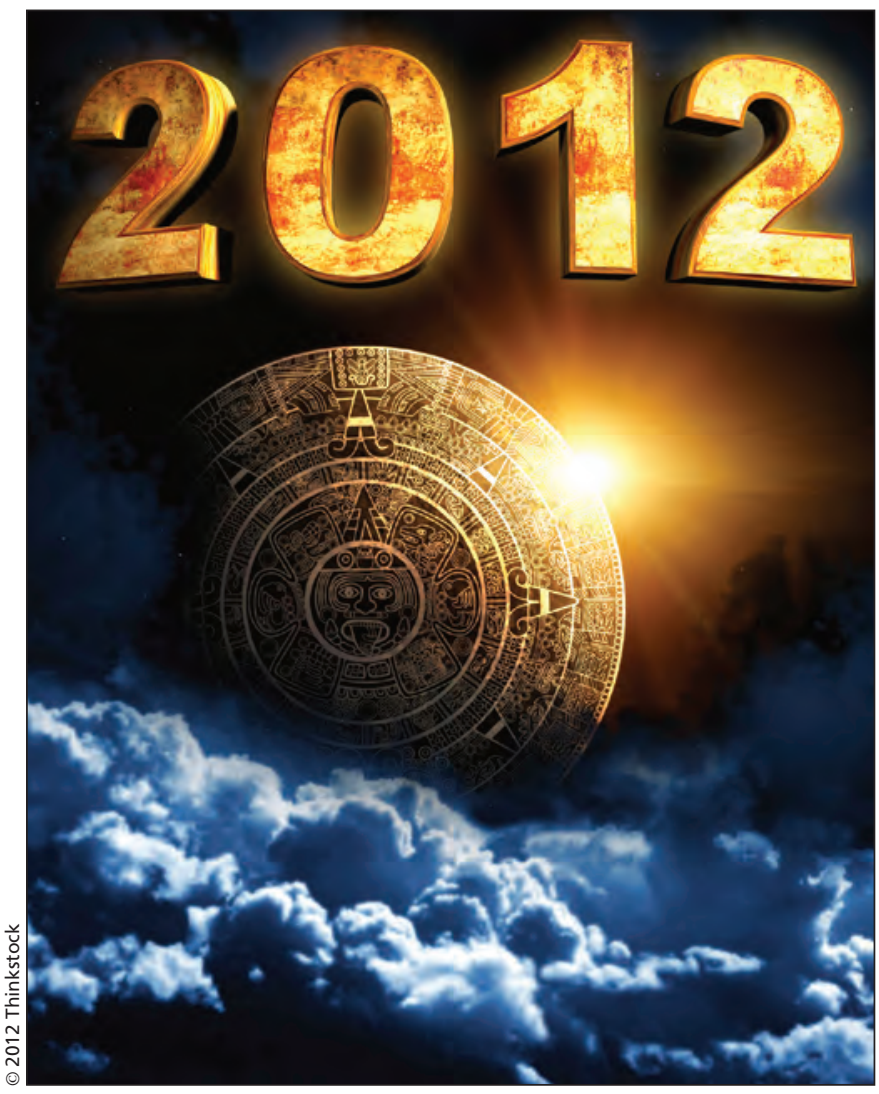

\section{Stuff we found out}

The difference in survival between the control and obliteration groups is shown in Figure 1. For the control group, death occurs at a predictable and fairly uniform rate. However, $\mathrm{MaD}$ leads to a statistically significant, and clinically relevant, difference in survival between the control and obliteration groups (we're pretty sure that, were it calculated, $p$ would definitely be something really significant, and certainly less than 0.05). Oddly, despite censoring for major known sources of bias (e.g., astronauts currently aboard the international space station, as well as zombies, the undead, the Grateful Dead, Dungeons and Dragons players, men who have read Fifty Shades of Grey and other similar beings likely 


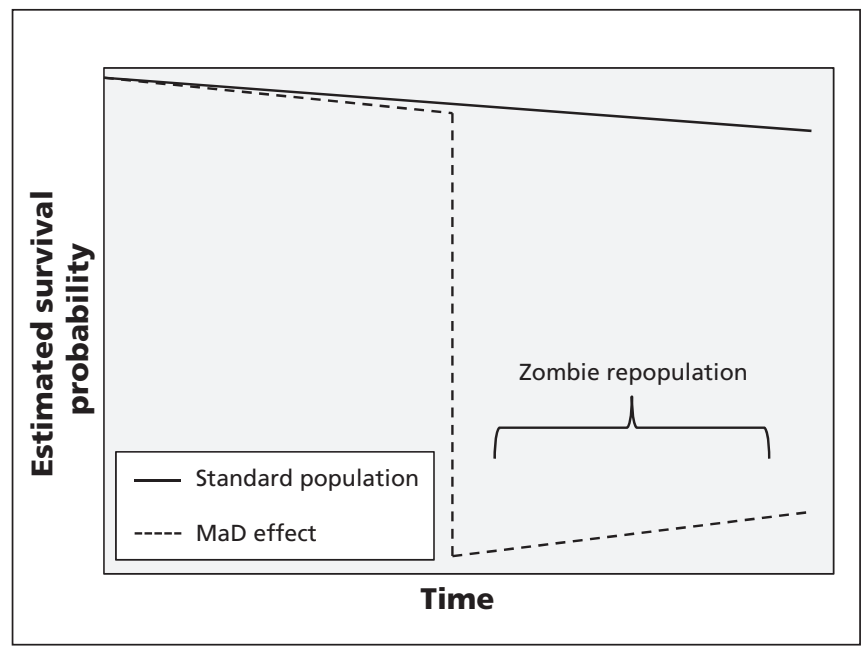

Figure 1: Survival curves for the overall population with and without the occurrence of Mayan doomsday. MaD = Mayan doomsday. Note heretofore unforeseen postapocalyptic zombie repopulation.

to be unaffected by the apocalypse), the obliteration group does not fall to 0 . We have dubbed this slow rise in the obliteration curve the "zombie repopulation."

Additional modelling was done to assess the effects of $\mathrm{MaD}$ on a sample clinical trial comparing the efficacy of 2 fictional, although completely plausible, drugs: Toxico and Horribilum. As can be seen in Figure 2A, compared with patients receiving Horribilum, those receiving Toxico had longer median overall survival. When the effect of $\mathrm{MaD}$ is added to the model (Figure 2B), no difference in survival can be seen between the 2 drugs. Furthermore, any adverse events would not be able to be recorded owing to "the mother of all adverse events," and any statistical significance between study arms would be lost.

\section{Chit chat}

If we have been thinking clearly, then it is apparent that the end of the world will have catastrophic effects on statistical analyses of survival outcomes. We therefore recommend that all clinical trials should stop immediately, as $\mathrm{MaD}$ will negate all potential trial results.

However, are trials such as those between our two fictional drugs entirely redundant in the event of $\mathrm{MaD}$ ? It is possible that some useful scientific data could be salvaged. Late effects of these drugs could potentially be studied during the zombie repopulation, assuming that the original consent forms would still be considered valid (surely folks on the ethics board will have survived). Unfortunately, this work may be pragmatically challenging, as we suspect that most members of research teams may themselves be obliterated. (Due to sheer misfortune, all of the authors of this paper missed the biannual "Defense Against Zombies" seminar held in our institution.)

The possibility of a zombie repopulation is an unexpected finding in the event of $\mathrm{MaD}$. Before $\mathrm{MaD}$, the walking dead were most commonly relegated to young adult novels and cable specialty channels and were not observable with standard scientific techniques. Their putative existence does, how-

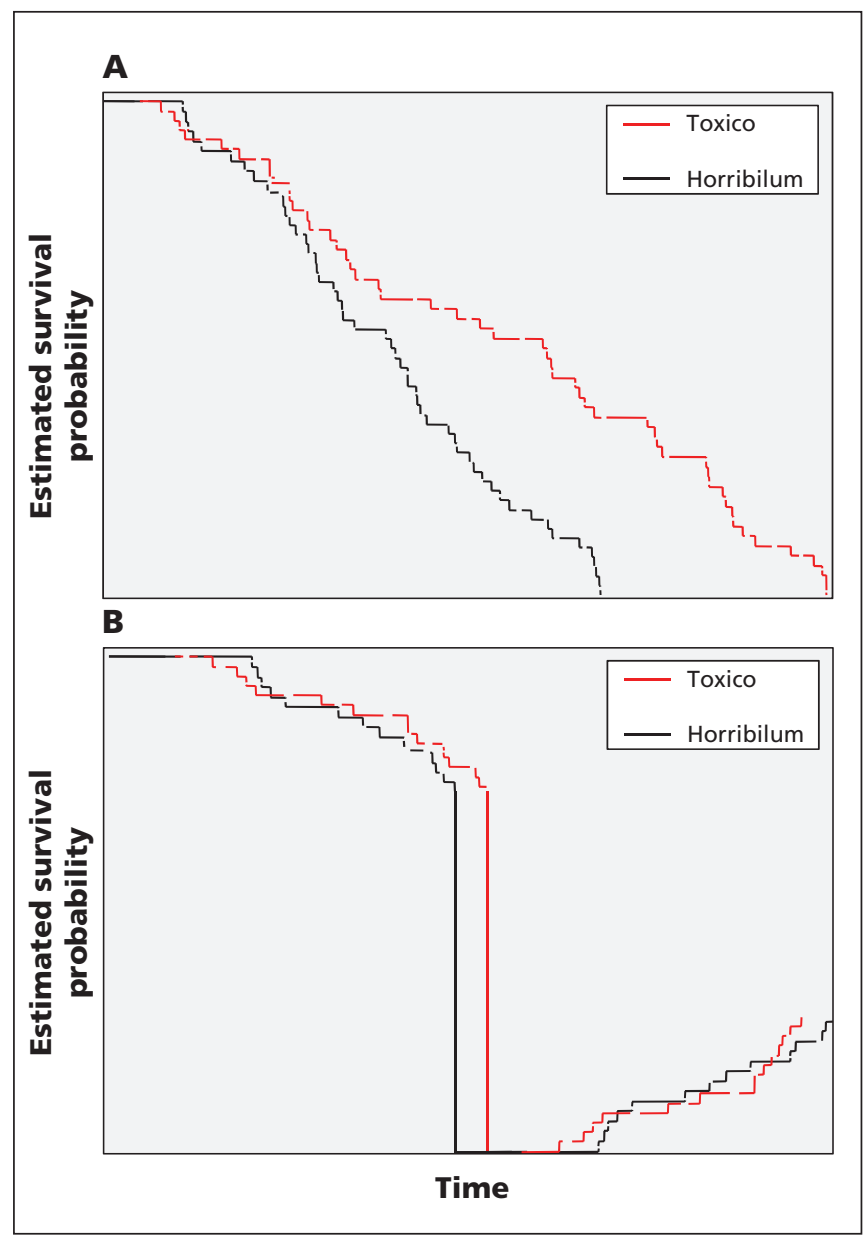

Figure 2: Kaplan-Meier curves comparing overall survival among patients receiving Toxico or Horribilum. (A) Results without the impact of Mayan doomsay. (B) Results with the impact of Mayan doomsday.

ever, bring a new dimension to the recent opening of multiple survivorship centres - although, if the new world population is predominantly immortal, we have concerns for the capacity of such programs. However, we would never allow such practical considerations to impair the scientific process.

\section{Conclusion}

$\mathrm{MaD}$ is bad.

\section{References}

1. 2012 phenomenon. Wikipedia. Available: http://en.wikipedia.org/wiki/2012_phenomenon (accessed 2012 Sept. 18)

2. MacDonald GJ. Does Maya calendar predict 2012 apocalypse? McLean (VA): USA Today; 2007. Available: http://usatoday30.usatoday.com/tech/science/200703-27-maya-2012_n.htm (accessed 2012 Sept. 18).

3. Vijayananthan A, Nawawi O. The importance of good clinical practice guidelines and its role in clinical trials. Biomed Imaging Interv J 2008;4:e5.

\section{Correspondence to: Paul Wheatley-Price, pwheatleyprice@toh.on.ca}

Affiliations: Paul Wheatley-Price and Mark Clemons are from the Department of Medicine, Division of Medical Oncology. Brian Hutton is with the Ottawa Health Research Institute Methods Centre, University of Ottawa, Ottawa, Ont.

CMAJ 2012. DOI:10.1503/cmaj.121616 